\title{
Research on the Inversion Method of the Source Item of the Battlefield Chemical Hazard Based on Genetic Algorithm
}

\author{
Jin $\mathrm{Gu}^{1, \mathrm{a}}$, Xuezheng Zhu ${ }^{2, \mathrm{~b}}$, Shunhua Liu ${ }^{1, \mathrm{c}}$, Xiaobin Liü \\ ${ }^{1}$ Department of Graduate Management, Institute of Chemical Defense, Beijing, 102205, China \\ ${ }^{2}$ Department of Chemical Defense Equipment, Institute of Chemical Defense, Beijing, 102205, \\ China \\ ${ }^{3}$ Unit 71320, Kaifeng, 475000, China

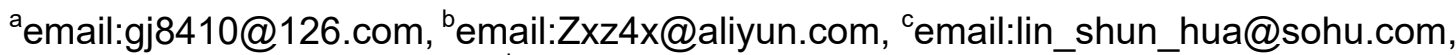 \\ demail:873963446@qq.com
}

Keywords: chemical hazard; source item; inversion; genetic algorithm

\begin{abstract}
Adopting the genetic algorithm, this paper studies the method of source term inversion through the data from chemical monitoring devices distributed in battlefield when we can not accurately judge the hazard source item data after the occurrence of chemical hazards in battlefield. Based on the data generated by Gauss diffusion model, the author verifies the inversion algorithm through the simulation data. The results show that the inversion algorithm can accurately estimate the source item information, which is important for the commanders to grasp and predict the diffusion trend of chemical hazards.
\end{abstract}

\section{Introduction}

After the chemical attack on the battlefield, the accurate prediction of the diffusion trend and range of chemical cloud will play an important role in the decision-making of the commander. At present, the prediction of battlefield chemical hazards is mainly conducted by numerical simulation of diffusion model of various chemical clouds, so as to define the hazardous areas. However, in the actual situation, it is difficult to improve the accuracy of prediction model, since there's a lot of uncertainty about the information of source strength, attack mode, and wind field of the chemical attack on the battlefield. Taking the Gauss diffusion model as an example, this paper studies how to use genetic algorithm to invert the source item data, so as to integrate the sensor data with diffusion model data and achieve the purpose of model optimization.

\section{Problem Descriptions}

The source item inversion is a method that uses the monitoring values around the source item to backward reason the source item's own parameters ${ }^{[1]}$. The source item inversion of chemical hazard in the battlefield just integrates the measurement data, location information, and meteorological data of each monitoring point through the various monitoring equipment distributed in the battlefield and then combines with the atmospheric dispersion model, in order to calculate the source item parameters in the occurrence of chemical hazards including source strength and position according to mathematical algorithm.

The estimation method of source item of traditional battlefield chemical hazards is carried out according to the attack way and attack scale of the attacked region, which needs to grasp the type and quantity of weapons in the chemical attack of the enemy. Next, the source strength will be estimated according to the smoke status of our side after attack. However, this method is affected greatly by the weather, terrain, and the attack way and the error of estimated results is relatively large. This paper uses the chemical monitoring equipment distributed in battlefield to estimate the source item according to the monitoring values of each position under the wind after the attack, which can effectively improve the accuracy of source item. 


\section{Model Building}

\section{Atmospheric Dispersion Model}

The atmospheric dispersion model is an important means to study the diffusion rule of chemical hazards in the air and also the mathematical calculation method to conduct quantitative analysis of transport and diffusion characteristics of chemical hazards in the atmosphere. Due to the complexity of the meteorological conditions, topography, and pollution that will affect the dispersion process, we have not been able to find an atmospheric dispersion model that is applicable to all conditions to describe all the atmospheric dispersion problems. The mathematical methods adopted by those models may be basically divided into the Gauss model and its deformation model, statistical model, similar model of atmospheric pressure diffusion, and K model.

Due to the short time of battlefield chemical hazard, we must judge the hazards situation under the wind in a very short time, so the Gauss model is usually adopted. The Gauss model needs less meteorologic conditions, calculation amount, and computing time. Under the dispersion conditions of constant atmospheric condition and uniform terrain level, the simulation results are reasonable, so it is a classical method of atmospheric dispersion. The Gauss diffusion equation is exported under the assumption that the poison cloud concentration is in compliance with the normal distribution. Its theoretical basis is that from the statistical theory, the probability distribution of displacement of diffusing particles is the normal distribution under the assumption of smooth and uniform turbulence.

In a stable and balanced atmosphere, we may suppose that the source strength $Q_{p}(g / s)$ from the origin of coordinate continuously and evenly casts aerosol agent into the atmosphere while the cast materials form the conical chemical clouds under the wind along the $\mathrm{X}$ axis direction in the battlefield space. We may imagine that the concentration of any point in the space will not change over time and the distribution of concentration at $x$ vertical section should be in accordance with two-dimensional normal distribution. As for the chemical hazards from the ground, the concentration expression $^{[3]}$ :

$$
C_{(x, y, z)}=\frac{Q_{p}}{\pi u \sigma_{x} \sigma_{y}} e^{-\left(\frac{y^{2}}{2 \sigma_{y}^{2}}+\frac{z^{2}}{2 \sigma_{z}^{2}}\right)}
$$

In the expression, $C_{(x, y, z)}$ is the concentration of any coordinate point, $Q_{p}$ is the continuous point source strength, and $\sigma_{x} \sigma_{y}$ is the atmospheric diffusion coefficient.

\section{Model of Genetic Algorithm}

Being founded in 1970s by the American professor J.H.Holland of the University of Michigan, the genetic algorithm (GA) is a kind of random search algorithm based on biological natural selection and genetic mechanism and also the adaptive probability optimization algorithm for complex system optimization. It is an important way to solve the general optimization problems by using the genetic algorithm coding and population propagation mechanism to express complex problems. Due to the large search space of genetic algorithm, the principle of genetic algorithm is simple in terms of complex problems and nonlinear solution space and there is no a priori requirement of results for the system to be optimized. These features are in accord with the characteristics of the source item inversion of the battlefield chemical hazard. The solution process can be described as follows in Figure 1.

(1) To determine the relevant parameters, including the population individual number $M$, hybrid probability $p_{\mathrm{c}}$, mutation probability $p_{\mathrm{m}}$, maximum number of evolution $\mathrm{N}$, and end conditions.

(2) The initial population individuals for generation of GA and SA. For the inversion of the source item of battlefield chemical hazard, the individual of the population is the source item, including the source strength $\mathrm{Q}$ and release position coordinate $\mathrm{A}(x, y)$.

(3) To calculate the fitness of each individual in the population. The fitness function is the general difference between the concentration $C_{i}$ of the actual monitoring point and the calculated 
value $R_{i}$, namely $f_{i}=\sqrt{\sum_{i=1}^{n}\left[\ln \left(C_{i}\right)-\ln \left(R_{i}\right)\right]^{2}} \quad / \sqrt{\sum_{i=1}^{n}\left[\ln \left(R_{i}\right)\right]^{2}}$

(4) To calculate the fitness $f_{i}^{*}$ of each individual in the new population and determine whether to meet the requirements of the termination conditions.

(5) To conduct the selection, crossover, and mutation operation in accordance with established rules so as to generate a new population.

(6) To return to $(3)^{[4]}$.

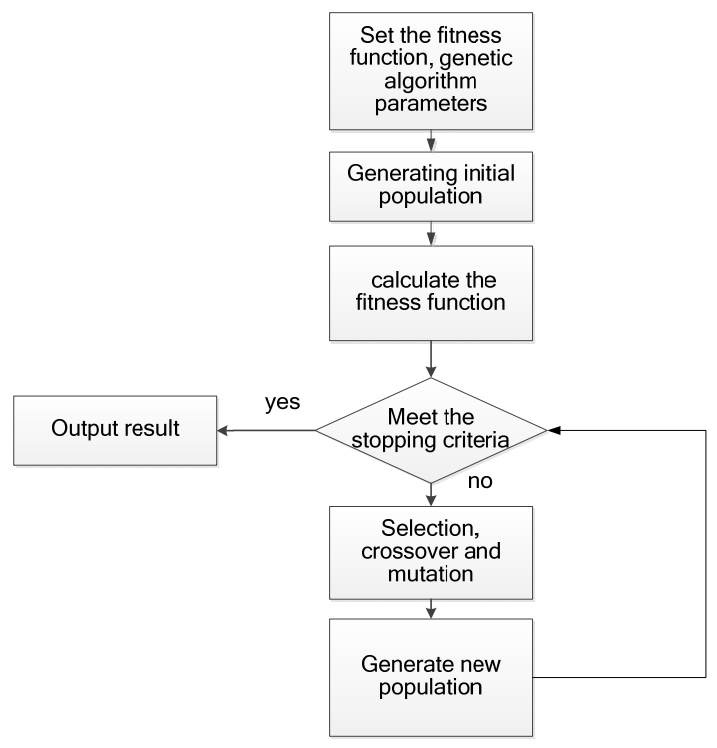

Figure 1 Solving Process of Genetic Algorithm

\section{Algorithm Design}

We may suppose that the chemical attack in battlefield happens at $\mathrm{t}=0$, the chemical hazardous substances release in the form of continuous point source, the release rate $Q_{0}=5000 \mathrm{~g} / \mathrm{s}$, the coordinates are $\mathrm{x}_{0}=0, \mathrm{y}_{0}=0$, and the wind direction is east. There are $8 \times 8$ gas monitoring devices distributed in the battlefield area evenly to obtain the concentration of the chemical hazardous substances in the location. Its distribution is shown in Figure 2.

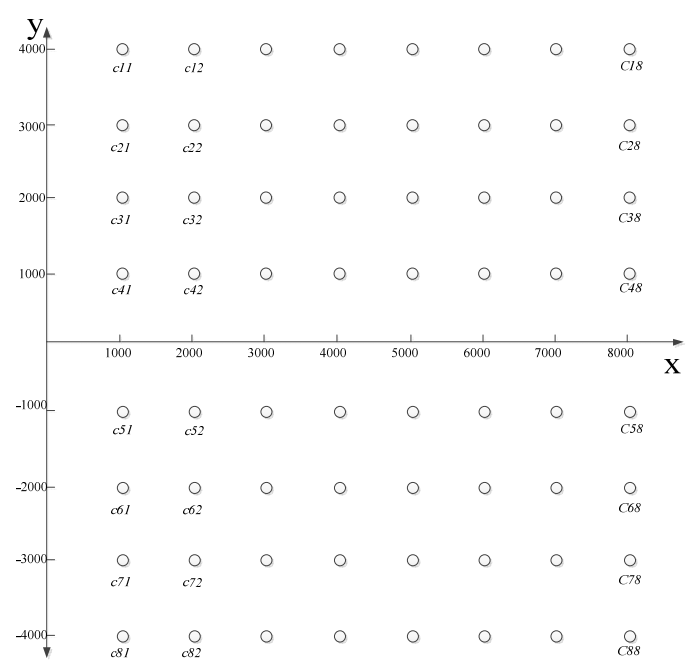

Figure 2 Distribution of Battlefield Chemical Hazards Monitoring Device

The pollution concentration distribution of chemical hazards in the battlefield is in accordance with Gauss's diffusion law. According to formula (1), we may calculate the theoretical strengths $c_{11} \sim C_{88}$ of each monitoring position in battlefield and take them as the model input values. The 
diffusion coefficient $\sigma_{x} \sigma_{y}$ in the model may be obtained according to Pasikuier's calculation method. We suppose that the stability level is E, and then:

$$
\begin{gathered}
\sigma_{y}=\gamma_{1} x^{\alpha^{1}}=0.9048 x^{0.0961} \\
\sigma_{z}=\frac{a x}{(1+b x)^{c}}=\frac{0.037 x}{(1+0.0015 x)^{0.57}}
\end{gathered}
$$

In the inversion model, the theoretical concentration of the monitoring point is used as the hypothetical actual concentration and retrieve the source term data based on genetic algorithm. Next, compared with the given input values, the error of the results can be analyzed as shown in Figure 3.

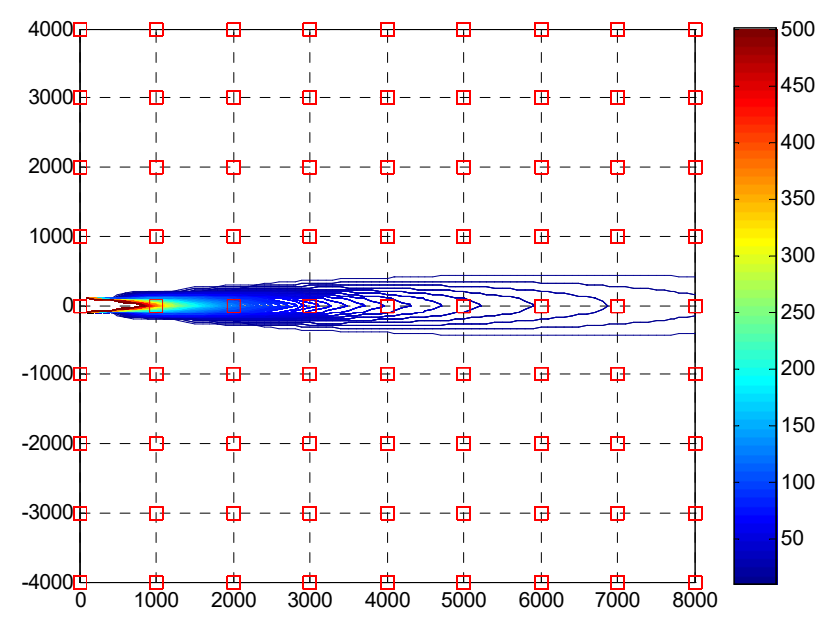

Figure 3 Schematic Diagram of Measured Point of Chemical Hazard Concentration in Battlefield

In this paper, the genetic algorithm toolbox of MATLAB is used to realize the source item inversion. There are the graphical user interface and command line functions, which can be used to describe problems, set algorithm options, and monitor algorithm process quickly. The genetic algorithm tool of multiple options in Matlab genetic algorithm toolbox can be used for problem creation, fitness calculation, selection, crossover, and mutation, which can meet the needs of this article.

\section{Results and Discussions}

We may substitute the above parameters into genetic algorithm in order to conduct the source item inversion. The parameter setting of genetic algorithm is: population number 20, hybrid probability 0.8 , selection method roulette, and iteration times 100 . The other parameters adopt the default parameters of the toolbox. In the genetic algorithm set up in this paper, the chemical pollution source $\mathrm{Q}(\mathrm{g} / \mathrm{s})$, the horizontal ordinate $x(\mathrm{~m})$, and longitudinal coordinate $\mathrm{y}(\mathrm{m})$ are the three parameters that need to be retrieved. Under the initial conditions, the search areas of the three parameters are set as Q:[0,10000], $\mathrm{x}:[-5000,5000], \mathrm{y}:[-5000,5000]$. The results of the 5 inversions are shown in Table 1. The variation of the fitness function value of the 5 groups with the number of iterations is shown in Figure 4.

In order to reflect the accuracy of the inversion results, the source inversion error function $\Delta Q=\frac{\left|Q-Q_{0}\right|}{Q_{0}} \times 100 \%$ is introduced and the function of source coordinate position error is $\Delta A=\frac{\sqrt{\left(x-x_{0}\right)^{2}+\left(y-y_{0}\right)^{2}}}{\sqrt{x^{2}{ }_{\text {max }}+y^{2}{ }_{\text {max }}}}$. In the formula, $x_{\max }$ is the maximum of $x$ search area while $y_{\max }$ is the maximum of $y$ search area. 

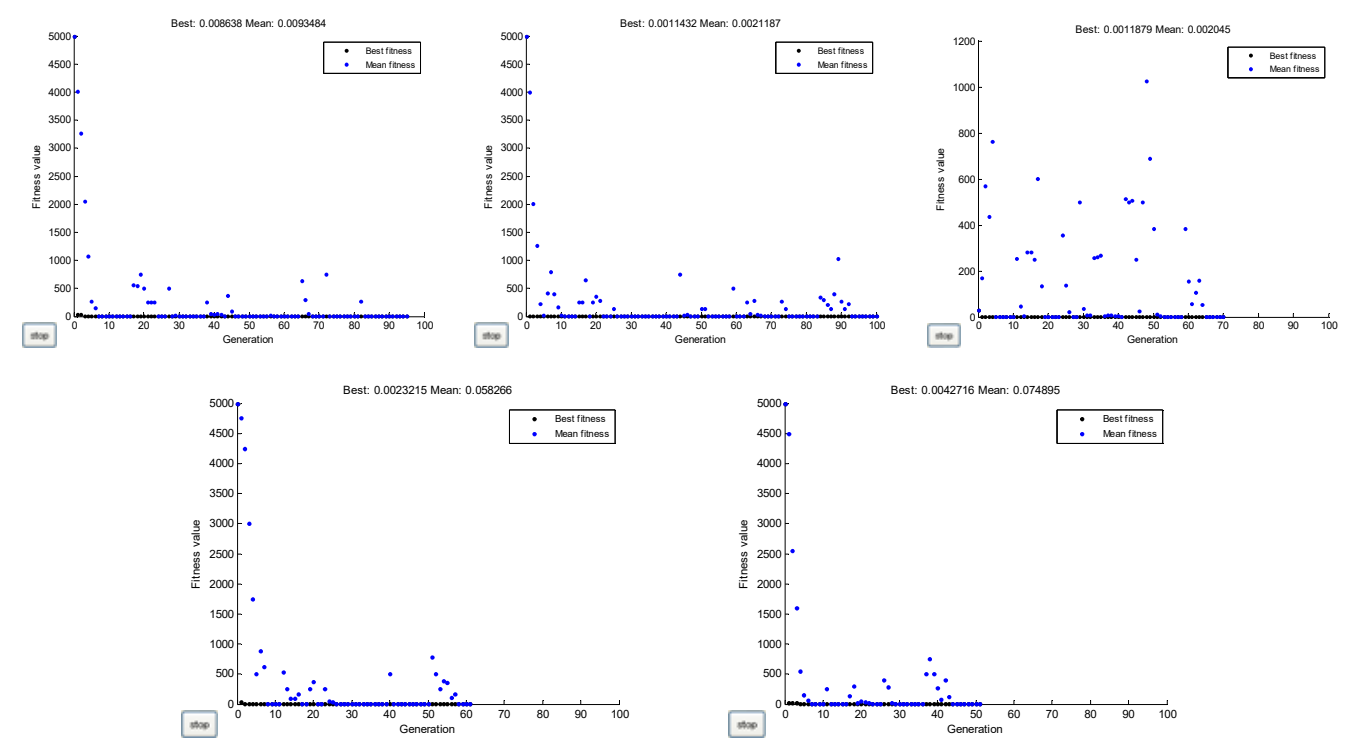

Figure 4 The Change Curve of Fitness Function Value

Table 1 Inversion Results

\begin{tabular}{|c|c|c|c|c|c|}
\hline Group & $\mathrm{Q}$ & $\mathrm{x}$ & $\mathrm{y}$ & $\Delta Q / \%$ & $\Delta A / \%$ \\
\hline 1 & 5025 & -1.832 & -0.2056 & 0.55 & 0.0206 \\
\hline 2 & 4887 & -13.99 & 3.132 & 2.26 & 0.1603 \\
\hline 3 & 4971 & -2.935 & 0.8156 & 0.58 & 0.0341 \\
\hline 4 & 5021 & -1.202 & -0.01979 & 0.42 & 0.0134 \\
\hline 5 & 5025 & -1.533 & -0.7427 & 0.50 & 0.019 \\
\hline
\end{tabular}

From the inversion results, a good convergence effect is achieved when the convergence rate of fitness function is fast and the iterations is 20 . By comparing the results of the 5 inversions, we find that the source error of inversion is less than $3 \%$ and the position error is less than $1 \%$, which shows that it is feasible to inverse the source term of the battlefield chemical hazards by genetic algorithm. However, the inversion algorithm in this paper is carried out under the ideal conditions.

The algorithm needs to be further improved if taking into account of the error, misinformation, and distribution of chemical sensors in battlefield.

\section{References}

[1] Yongsheng Ling, Wenyu Hou, Wenbao Jia. Research on the Inversion Method of Nuclear Accident Source Item Based on BP Neural Network[J], Chinese Journal of Safety Science, 2014, 24(7):21-25

[2] Wensheng Niu, Zhenhai Sun. A Brief Review of the Atmospheric Dispersion Model [J], Meteorological Science and Technology, 2000,(2):1-4

[3] Jinzhou Chen, Haiping Chen. Effects and Destruction of Chemical Weapon [M], Weapon Industry Press, 2002

[4] Ying Zhang, Manchun Liang, Ke Li. Research on Source Item Inversion Method Based on Genetic Simulated Annealing Algorithm [J]. Nuclear Electronics \& Detection Technology, 2014, 34(4):451-455

[5] Jike Ge, Yuhui Qiu, Chunming Wu. Summary of Genetic Algorithm Research [J]. Computer Application Research, 2008, 25(10):37-42 